\title{
The impact and cost-effectiveness of 9-valent human papillomavirus vaccine in adolescent females in Hong Kong
}

Tak Hong Cheung ${ }^{1 \dagger}$, Sally Shuk Yee Cheng ${ }^{2 \dagger}$, Danny C. Hsu², Queenie Wing-Lei Wong ${ }^{2}$, Andrew Pavelyev ${ }^{3,4}$, Anuj Walia ${ }^{3}$, Kunal Saxena ${ }^{3}$ and Vimalanand S. Prabhu ${ }^{3^{*}}$

\begin{abstract}
Introduction: In Hong Kong (HK), a single-cohort vaccination program for 10-12-year-old girls with the 9-valent human papillomavirus (HPV) vaccine (9vHPV; types 6/11/16/18/31/33/45/52/58) has been launched. This study assessed the public health impact and cost-effectiveness of implementing routine 9vHPV vaccination (12-year-olds) with or without catch-up 9vHPV vaccination (13-18-year-olds) in HK.
\end{abstract}

Methods: The health impact and costs of implementing routine 9vHPV vaccination with or without catch-up vaccination over a 100-year time horizon were evaluated using a validated HPV-type transmission dynamic model adapted to the HK population; analyses were performed from a healthcare payer perspective. Routine vaccination (12-year-old girls) and catch-up vaccination (13-18 years) assumed vaccine coverage rates of 70\% (base case) and 30\%, respectively. The model also assumed herd immunity, lifelong vaccine protection, a discount rate of 3\%, and a cost per dose of HK dollars (HKD) 858 [United States dollars (USD) 110] and HKD 1390 (USD 179) for the 2-valent HPV (2VHPV) and $9 \mathrm{VHPV}$ vaccines, respectively. HPV disease-related incidence and the incremental cost-effectiveness ratio (ICER) per quality-adjusted-life-year (QALY) were estimated. Cost-effectiveness was determined at a ceiling threshold of HK dollars (HKD) 382,046 (USD 49,142) or 1.0 times the gross domestic product per capita of HK.

Results: Compared with routine 9vHPV alone, routine plus catch-up 9vHPV is projected to reduce cervical cancer incidence by 3.4\%. Routine plus catch-up 9vHPV will also reduce genital warts incident cases for males/females by 2.6\%/5.4\%. The incremental cost-effectiveness ratios were HKD 29,911 (USD 3847)/quality-adjusted life-year (QALY) for routine plus catch-up 9vHPV versus routine 9vHPV alone and HKD 25,524 (USD 3283)/QALY for routine 9vHPV alone versus screening only. Sensitivity analyses indicated that routine plus catch-up 9vHPV compared with routine 9vHPV alone remained cost-effective at coverage rates of 30\% and 90\%.

Conclusions: This analysis predicts that the current HK vaccination strategy can be considered cost-effective and will provide maximum health benefit. These results support addition of the routine 9vHPV vaccine with or without catchup 9vHPV vaccination to the regional vaccination program in HK.

Keywords: Cervical cancer, Cost-effectiveness, Genital warts, Hong Kong, Human papillomavirus, Vaccine

*Correspondence: vimalanand.prabhu@merck.com

${ }^{\dagger}$ Tak Hong Cheung and Sally Shuk Yee Cheng contributed equally to the manuscript

${ }^{3}$ MRL, Merck \& Co., Inc., 2000 Galloping Hill Road, Kenilworth, NJ 07033, USA

Full list of author information is available at the end of the article

\section{Background}

Human papillomavirus (HPV) infection causes almost all cases of cervical cancer and is relevant in other cancers, including anal, vulvar, vaginal, penile, and head and neck [1]. These cancers are typically a result of infection with original author(s) and the source, provide a link to the Creative Commons licence, and indicate if changes were made. The images or other third party material in this article are included in the article's Creative Commons licence, unless indicated otherwise in a credit line to the material. If material is not included in the article's Creative Commons licence and your intended use is not permitted by statutory regulation or exceeds the permitted use, you will need to obtain permission directly from the copyright holder. To view a copy of this licence, visit http://creativecommons.org/licenses/by/4.0/. The Creative Commons Public Domain Dedication waiver (http://creativeco mmons.org/publicdomain/zero/1.0/) applies to the data made available in this article, unless otherwise stated in a credit line to the data. 
high-risk oncogenic HPV 16/18/31/33/35/39/45/51/52/5 6/58/66/68/70, whereas nononcogenic HPV types, most frequently HPV 6/11, are associated with genital warts and recurrent respiratory papillomatosis [2].

Cervical cancer is the ninth most common cancer and the ninth leading cause of cancer-related deaths among females in Hong Kong, with crude and age-standardized incidence rates of 12.9 per 100,000 females and 7.6 per 100,000 females, respectively [3]. The overall genital warts incidence rate in Hong Kong is estimated at 203.7 per 100,000 person-years and is higher in males than in females (292.2 vs 124.9 per 100,000 person-years) [4].

Worldwide, the five most prevalent types associated with cervical cancer are HPV 16/18/45/31/33, although geographic variations exist [5]. In Hong Kong, the four most prevalent HPV types associated with cervical cancer are HPV 16/18/52/58 [6]. A study in Hong Kong found HPV 16/52/58 as the primary HPV types attributed to squamous cell carcinoma and cervical intraepithelial neoplasia (CIN)-1/2/3 lesions, indicating that an HPV vaccine that protects against HPV types $52 / 58$ is projected to increase coverage of $11.7 \%$ of cervical cancers, $17.7 \%$ for CIN-1 lesions, $22.6 \%$ for CIN-2 lesions, and $14.4 \%$ for CIN-3 lesions, compared with an HPV vaccine that only covers HPV 16/18 [7].

There are currently two vaccines available for the prevention of HPV-related diseases on the market in Hong Kong. The 2-valent HPV (2vHPV) vaccine protects against HPV $16 / 18$, responsible for $70 \%$ of cervical cancer cases globally [8], whereas the 9-valent HPV (9vHPV) vaccine offers increased coverage against oncogenic HPV 16/18/31/33/45/52/58, which are associated with approximately $90 \%$ of cervical cancers [8], as well as nononcogenic HPV 6/11 associated with $90 \%$ of genital warts cases [9].

Both the World Health Organization and the Centre for Health Protection in Hong Kong recommend HPV vaccination along with regular screening for the prevention of cervical cancer [10, 11]. In 2004, a cervical cancer screening program was implemented in Hong Kong that recommends screening by cytology every 3 years following two consecutive normal annual smears in females aged $25-64$ years [12]. Although registration with the program increased from $3.6 \%$ in 2004 to $20.5 \%$ in 2017 , cervical Pap testing remained low (57\% between 2011 and 2014) [12]. Previous reports have also noted that without a government immunization program, HPV vaccine uptake rates are low in school-aged (11-18 years; approximately $2-9 \%)$ and university-aged ( $<26$ years; $9.7 \%)$ females [13-15]. Potential barriers to vaccination include the cost of vaccination and lack of access to information about the HPV vaccine [13, 14].
The Hong Kong government launched a vaccination program with the 9vHPV vaccine during the 2019/2020 school year in all eligible female primary school students in grades 5 and 6 . An interim target vaccine coverage of $70 \%$ for the 2-dose HPV vaccination schedule was set by the Hong Kong government, with preliminary data reporting a vaccine coverage rate of $85 \%$ for the first dose as of December 31, 2020 [16]. The decision to launch this HPV vaccination program was based on a modeling analysis demonstrating that 20 years of routine 9vHPV vaccination in adolescent females in Hong Kong is highly likely to be cost-effective, and the maximum cost for the vaccine to be cost-effective is sensitive to vaccine coverage [17]. These estimates are likely to be conservative as the analysis did not account for other HPV-related diseases (i.e., vulvar, vaginal, or anal cancers and genital warts) or catch-up vaccination [17]. 9vHPV vaccination of school-aged females (10-12-year-olds) has also been shown to be potentially cost-effective in other countries, including Canada and Singapore [18, 19]. A similar model-based analysis conducted for the population of Canada determined that vaccination of 10-year-old girls with the 9vHPV vaccine can be cost-effective, compared with vaccination with the 4-valent HPV (4vHPV) vaccine [18].

Given the high prevalence of HPV 52/58 in Hong Kong, the low historical coverage of HPV vaccination, and plans for the Hong Kong government to introduce a routine HPV vaccination program, there is a need to determine the public health and economic impact of school-based $9 \mathrm{vHPV}$ vaccination. We use a model to assess every next cohort at 12 year olds receiving the vaccination with continued coverage of 100 years. Therefore, the aim of this analysis was to assess the impact of a school-based strategy comprising two-dose 9vHPV routine vaccination of 12-year-old girls with or without catch-up 9vHPV vaccination among females aged 13-18 years (two-dose catchup among those aged 13-14 and three-dose catch-up with 9vHPV vaccination among those aged $\geq 15$ years) along with screening in the Hong Kong setting.

\section{Methods \\ Model design}

A previously validated HPV-type transmission dynamic mathematical model simulating the natural history of HPV infections and estimating the cost associated with HPV-related diseases [20] was adapted to the Hong Kong setting in order to evaluate the public health and economic impact of HPV vaccination with routine 9vHPV and routine plus catch-up 9vHPV. The current model comprises HPV infection and disease state transitions, lifetime duration of infection-derived immunity, and 
unvaccinated compartments, which have been previously described in detail [20].

\section{Target population, study perspective, and time horizon}

The target population for routine vaccination was 12-year-old girls, whereas catch-up vaccination was considered for females aged 13-18 years. The health impact was assessed for the entire Hong Kong population across all ages. Costs were assessed using a healthcare payer perspective and impact was measured over a 100-year time horizon because this was considered an appropriate time frame from which the system approached steady state and most benefits and costs of vaccination could be realized. The median age of diagnosis is 49 years for cervical cancer and 62-68 years for other HPV-associated cancers [21]. Given that the target age for routine vaccination in this analysis is 12 years, it may take a long period of time before the benefits of HPV vaccination on costs and outcomes become apparent. Herd immunity was projected over the total population of Hong Kong (7.3 million people) [22] over a 100 -year time period in the transmission model.

\section{Scenarios compared}

The vaccination scenarios compared include: (1) screening only, (2) routine 9vHPV vaccination (routine 9vHPV) along with screening, and (3) routine plus catch-up 9vHPV vaccination (routine plus catch-up 9vHPV) along with screening. The focus of this paper will be on routine 9vHPV vaccination with or without catch-up 9vHPV vaccination along with screening.

\section{Model compartments}

The age-structured mathematical model [20] is a single dynamic model that incorporates epidemiology of HPV infection, disease, and economics. Therefore, both the direct and indirect herd immunity benefits and costs of $\mathrm{HPV}$ vaccination can be captured by the model for the population over time in a transparent and reproducible manner.

The model structure, equations used, and assumptions have been previously described in detail [20]. At an assumed age of sexual debut of 12 years, individuals enter the population at a sex-specific and sexual-activityspecific rate, moving between successive age groups at an age- and sex-specific rate per year, and exit upon death at an age- and sex-specific per capita death rate per year. An additional age- and stage-dependent death rate is applied for patients with cervical cancer; CIN and genital warts are assumed to carry no additional risk of death. The agestructured model also simulated HPV transmission and the occurrence of CIN, cervical cancer, and genital warts. The acquisition of an infection and progression to disease follow a similar natural history structure, as assumed in previous models for HPV 16/18 [23]. Within the population, patients were divided into epidemiologic categories based on their infection, disease, screening, and treatment status.

The model comprised three connected modules: (1) a demographic variables model describing the age structure of the population (i.e., birth, aging, and death) and behavioral variables describing sexual activity; (2) an epidemiologic model simulating the transmission of HPV $6 / 11 / 16 / 18 / 31 / 33 / 45 / 52 / 58$, occurrence of genital warts, precancers such as CIN $1 / 2 / 3$ and vaccine type-related cervical cancer; and (3) a disease variables model describing screening rates, HPV infection, and HPV-related diseases [20].

\section{Health outcomes and economic outputs}

The mathematical model simulated and tracked various outcomes, including numbers of cases of precancers (i.e., CIN-1, CIN-2, CIN-3, vaginal intraepithelial neoplasia $2 / 3$ ), cervical cancer, vaginal/vulvar cancer, and anal cancer. The model also estimated quality-adjusted life years (QALYs) and total costs, including vaccine-preventable disease costs, and vaccination costs. The incremental cost-effectiveness ratio (ICER) was estimated as the ratio of incremental cost to incremental QALYs. Cost-effectiveness was determined at a ceiling threshold of Hong Kong dollars (HKD) 382,046 [US dollars (USD) 49,142] or 1.0 times the gross domestic product per capita of Hong Kong; this threshold reflected the maximum willingness of decision-makers to pay for an additional QALY [24].

\section{Inputs}

Variables input into the model are shown in Table 1 and Additional file 1: Table S1. Input costs were derived from prices charged to a private case in a public hospital in Hong Kong (Data on file). It is assumed that the private charges in a public hospital setting are equivalent to the cost incurred to the public healthcare provider of the government of Hong Kong.

HPV vaccination coverage varies by country. Given that this would be a novel program in Hong Kong, we assumed a $70 \%$ coverage in 12-year-old girls for routine vaccination for the base case. We assumed 30\% coverage in females aged 13-18 years for catch-up vaccination.

The model considered oncogenic HPV $16 / 18 / 31 / 33 / 45 / 52 / 58$ for cervical cancer, anal cancer, and CIN $1 / 2 / 3$, as well as nononcogenic HPV 6/11 for genital warts. For all other HPV-related diseases (i.e., vaginal cancer, vulvar cancer, and vaginal intraepithelial neoplasia 2/3), the model only considered HPV 16/18.

The model also assumed herd immunity, lifelong duration of vaccine protection, a discount rate of $3 \%$ for costs 
Table 1 Model input parameters

\begin{tabular}{|c|c|c|c|}
\hline \multicolumn{3}{|l|}{ Input parameter } & \multirow[t]{2}{*}{ Data source } \\
\hline \multicolumn{3}{|l|}{ Demographic variables } & \\
\hline Total population size (males and females), 2016 & \multicolumn{2}{|l|}{$7,336,600$} & \multirow[t]{2}{*}{ Hong Kong Census [22] } \\
\hline \multicolumn{3}{|l|}{ Sexual behavior variables } & \\
\hline \multicolumn{4}{|c|}{ Percentage of the population in the following sexual activity risk groups } \\
\hline Sexual activity category & Males & Females & \multirow[t]{4}{*}{ Youth Sexuality Study 2011 [27] } \\
\hline Low (0-1 mean sexual partners per year) & 80 & 94 & \\
\hline Medium (2-4 mean sexual partners per year) & 15 & 5 & \\
\hline High ( $\geq 5$ mean sexual partners per year) & 5 & 1 & \\
\hline \multicolumn{4}{|c|}{ Mean number of sexual partners per year by the following sexual activity risk groups } \\
\hline Sexual activity category & Males & Females & \multirow[t]{4}{*}{ Youth Sexuality Study 2011 [27] } \\
\hline Low (0-1 mean sexual partners per year) & 0.75 & 0.92 & \\
\hline Medium (2-4 mean sexual partners per year) & 2.52 & 2.43 & \\
\hline Medium (2-4 mean sexual partners per year) & 6.1 & 7.14 & \\
\hline Screening variables & & & $\begin{array}{l}\text { Hong Kong Department of Health Cervical } \\
\text { Screening Programme } 2015 \text { [45] }\end{array}$ \\
\hline \multicolumn{4}{|l|}{ Disease variables } \\
\hline Cervical cancer & & & $\begin{array}{l}\text { Hong Kong Cancer Registry } 2015 \text { [25], Chan } \\
2009 \text { [46], Chan } 2011 \text { [7], Lin } 2010 \text { [4] }\end{array}$ \\
\hline Vaginal/vulvar cancer & & & $\begin{array}{l}\text { Hong Kong Cancer Registry } 2015 \text { [25], De Martel } \\
2017 \text { [8] }\end{array}$ \\
\hline Anal cancer & & & Hong Kong Hospital Authority data (Data on file) \\
\hline
\end{tabular}

and QALYs, and a cost per dose of HKD 858 (USD 110) and HKD 1390 (USD 179) for the 2vHPV and 9vHPV vaccines, respectively. The cost per dose includes the cost of the vaccine plus the administration fee.

\section{Inputs used for model calibration}

Inputs used to calibrate the model include population size (2016 data) [22]; incidence and mortality rates for cervical cancer [25], vaginal/vulvar cancer, 21 genital warts, and anal cancer; annual all-cause mortality rate by sex and age (2016 data) [26]; percentage attribution of cervical cancers of different HPV types; cervical cancer screening rate and age group; sexual behavior variables (including annual mean number of sexual partners by age group; percentage of the population at low, medium, and high sexual activity risk; and mean number of sexual partners per year by sexual activity risk group) [27]; hysterectomy rates by age group [22]; and costs associated with a single episode-of-care for genital warts, cervical cancer screening and visit, colposcopy, biopsy, CIN (1/2/3), cervical cancer, vaginal/ vulvar cancer, and anal cancer (Data on file). Additional inputs, such as CIN $1 / 2 / 3$ treatment variables, were derived from reports of private cases from the Hong Kong Hospital Authority (Data on file).

\section{Sensitivity analysis}

Deterministic one-way sensitivity analyses were conducted to assess the sensitivity of ICER values to variables that have been shown to be impactful to cost effectiveness. One variable was routine vaccination coverage, which was assessed at rates of $30 \%$ and $90 \%$. Rationales for selecting different coverage rates: (1) a rate of $30 \%$ was chosen, because the HPV catch-up program is more likely to be a public-private partnership with private clinics rather than school-based. As a result, a much lower vaccine uptake rate is expected, and (2) a rate of $90 \%$ was based on historical data showing that school-based programs of some vaccines (e.g., DTaP-IPV, MMR, and hepatitis B vaccines) can reach VCR as high as 90\% in Hong Kong [28]. Sensitivity analyses also assessed costs $( \pm 10 \%)$ and vaccine price discount rates (1\% and $5 \%)$.

\section{Results}

HPV-related disease incidence

Compared with routine 9vHPV alone, routine plus catchup 9vHPV is projected to prevent 769 (3.4\% reduction) cases of cervical cancers, over 100 years (Table 2). The model also demonstrated that, compared with routine 9vHPV alone, routine plus catch-up 9vHPV will avert 121 cases of CIN-1 (4.9\% reduction), 428 cases of CIN-2/ CIN-3 (4.6\% reduction), 6401 cases of genital warts 
among females (5.4\% reduction), and 10,090 cases of genital warts among males (2.6\% reduction) (Table 2$)$. The number of cases averted by routine plus catch-up 9vHPV and routine 9vHPV alone were both higher, compared with screening only (Table 2).

Compared with routine 9vHPV alone, routine plus catch-up 9vHPV will also result in 3.1\%, 3.4\%, and 3.0\% reductions in the incidences of vaginal cancer, vaginal intraepithelial neoplasia $2 / 3$, and vulvar cancer, respectively (Table 2).

Routine plus catch-up 9vHPV resulted in cumulative reductions in anal cancer incidence of $2.4 \%$ and $1.8 \%$ for females and males, respectively, compared with routine 9vHPV alone (55 and 42 cases avoided, respectively) (Table 2). Routine plus catch-up 9vHPV and routine 9vHPV alone will lead to a cumulative reduction in anal cancer incidence rates in both females and males (Table 2).

\section{Economic evaluation and cost-effectiveness}

Vaccine-preventable disease costs were reduced by $2.1 \%$ [approximately HKD 61.4 million (USD 7.9 million)] for routine plus catch-up 9vHPV versus routine 9vHPV alone (Table 2). Both routine plus catch-up 9vHPV and routine 9vHPV alone would also result in reductions in vaccine-preventable disease costs, compared with screening only (Table 2). Most of the vaccine-preventable disease-related cost reductions associated with routine plus catch-up 9vHPV were attributed to cervical cancer-related treatment costs [HKD 42.5 million (USD 5.5 million) saved]. Long-term trends in estimated healthcare costs avoided over 100 years for routine plus catchup 9vHPV versus routine 9vHPV alone indicated that most of the cost reductions were associated with HPV 16/18-related treatment costs (59.4\% of costs avoided) (Additional file 1: Fig. S1).

Screening-only resulted in the least QALYs, followed by routine $2 \mathrm{vHPV}$ alone, routine $9 \mathrm{vHPV}$ alone, and routine plus catch-up 9vHPV. ICERs for routine plus catch-up $9 \mathrm{vHPV}$ versus routine $9 \mathrm{vHPV}$ alone and routine $9 \mathrm{vHPV}$ alone versus screening only were HKD 29,911/QALY (USD 3847/QALY) and HKD 25,524/QALY (USD 3283/ QALY), respectively (Table 3; Additional file 1: Table S2).

\section{Sensitivity analysis}

Although cost-effectiveness was sensitive to vaccination coverage, routine vaccination with 9vHPV remained cost-effective at $30 \%$ and $90 \%$ coverage (ICERs: HKD 17,804/QALY [USD 2290/QALY] and HKD 29,434/QALY [USD 3786/QALY], respectively) compared with routine female-only vaccination with 2vHPV (Additional file 1: Table S3). Furthermore, routine plus catch-up 9vHPV compared with routine 9vHPV alone remained cost-effective at 30\%, 70\% (base case), and 90\% coverage [ICERS: HKD 19,979/ QALY (USD 2570/QALY), HKD 29,911/QALY (USD

Table 2 Reductions in HPV-related disease incidence for routine plus catch-up 9-valent HPV (9vHPV) female-only vaccination (FOV) vs routine 9vHPV FOV, routine 9vHPV FOV vs screening, and routine plus catch-up 9vHPV FOV vs screening, over 100 years

\begin{tabular}{|c|c|c|c|}
\hline HPV-related disease & $\begin{array}{l}\text { Reduction in cases of HPV-related } \\
\text { diseases } n(\%) \text { : Routine plus catch- } \\
\text { up } 9 \mathrm{vHPV} \text { vs routine } 9 \mathrm{vHPV}\end{array}$ & $\begin{array}{l}\text { Reduction in cases } \\
\text { of HPV-related disease } \\
\mathrm{n}(\%) \text { : Routine } 9 \mathrm{vHPV} \text { vs } \\
\text { screening only }\end{array}$ & $\begin{array}{l}\text { Reduction in cases } \\
\text { of HPV-related disease } \\
\text { n(\%): Routine plus catch- } \\
\text { Up } 9 \text { vHPV vs screening } \\
\text { only }\end{array}$ \\
\hline Cervical cancer & $769(3.4)$ & $19,880(46.9)$ & $20,649(48.7)$ \\
\hline CIN 1 & $121(4.9)$ & $3968(61.8)$ & $4089(63.7)$ \\
\hline $\mathrm{CIN} 2 / 3$ & $428(4.6)$ & $13,766(59.7)$ & $14,194(61.5)$ \\
\hline Vaginal cancer & $2(3.1)$ & $48(38.4)$ & $50(40.2)$ \\
\hline Vaginal intraepithelial neoplasia $2 / 3$ & $2(3.4)$ & $51(43.6)$ & $54(45.5)$ \\
\hline Vulvar cancer & $5(3.0)$ & $92(37.2)$ & $97(39.1)$ \\
\hline Genital warts (female) & $6401(5.4)$ & $306,673(72.3)$ & $313,074(73.8)$ \\
\hline Genital warts (male) & $10,090(2.6)$ & $534,895(58.2)$ & $544,985(59.3)$ \\
\hline HPV 6/1 1-related CIN 1 & $20(4.2)$ & $894(65.3)$ & $914(66.7)$ \\
\hline Anal cancer (female) & $55(2.4)$ & $1087(32.2)$ & $1142(33.8)$ \\
\hline Anal cancer (male) & $42(1.8)$ & $849(27.1)$ & $891(28.4)$ \\
\hline $\begin{array}{l}\text { Reduction in vaccine-preventable disease costs, } \\
\% \text { (HKD) }\end{array}$ & $61,366,272(2.1)$ & $884,053,918(23.2)$ & $945,420,190(24.8)$ \\
\hline
\end{tabular}

2vHPV 2-valent human papillomavirus, 9vHPV 9-valent human papillomavirus, CIN cervical intraepithelial neoplasia HKD Hong Kong dollars, HPV human papillomavirus

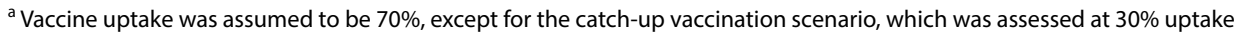


Table 3 Cost-effectiveness analysis of routine plus catch-up vs routine 9vHPV female-only vaccination strategies

\begin{tabular}{|c|c|c|c|c|c|}
\hline & \multicolumn{2}{|l|}{ Discounted total } & \multicolumn{3}{|l|}{ Incremental } \\
\hline & Costs/person (HKD) & QALYs/Person (y) & Costs/person (HKD) & QALYs/person (y) & $\begin{array}{l}\text { Costs/ } \\
\text { QALYs } \\
\text { (HKD/y) }\end{array}$ \\
\hline Routine 9vHPV FOV & 1855.97 & 27.25912 & NA & NA & NA \\
\hline $\begin{array}{l}\text { Routine plus catch-up 9vHPV } \\
\text { FOV }\end{array}$ & 1879.45 & 27.25990 & 23.48 & 0.000785 & $29,911^{\mathrm{a}}$ \\
\hline
\end{tabular}

9vHPV 9-valent human papillomavirus, FOV female-only vaccination, HKD Hong Kong dollar, ICER incremental cost-effectiveness ratio, NA not applicable, QALY qualityadjusted life-year

${ }^{a}$ Incremental costs, QALYs, and ICERS with respect to routine 9vHPV FOV

3847/QALY), and HKD 37,617/QALY (USD 4839/ QALY), respectively] (Additional file 1: Table S3). Routine 9vHPV alone compared with 2vHPV also demonstrated cost-effectiveness at $30 \%$ and $90 \%$ coverage [ICERS: HKD 17,804/QALY (USD 2290/QALY) and HKD 29,434/QALY (USD 3786/QALY), respectively]. Cost-effectiveness was also sensitive to vaccine discount rate for routine plus catch-up 9vHPV versus routine $9 \mathrm{vHPV}$ alone, with sensitivity analyses projecting an improvement in the ICER [HKD 5701/ QALY (USD 733/QALY)] when the discount rate was decreased to $1 \%$, whereas increasing the discount rate to 5\% will increase the ICER to HKD 84,648/QALY (USD 10,888/QALY) (Additional file 1: Table S3). Costeffectiveness was not sensitive to disease costs for routine plus catch-up 9vHPV versus routine 9vHPV alone, with sensitivity analyses projecting a small decrease in ICER when assuming costs + 10\% [HKD 28,764/QALY (USD 3700/QALY)] and a small increase when assuming costs - 10\% [HKD 31,043/QALY (USD 3993/ QALY)] (Additional file 1: Table S3). Similar trends were observed for routine 9vHPV alone versus routine $2 \mathrm{vHPV}$ alone with regards to sensitivity analyses for discount rate (1\% and $5 \%)$ and costs $( \pm 10 \%)$ (Additional file 1: Table S3).

The current analysis evaluated the impact of HPV vaccination with no change in screening patterns. Most models do not change screening patterns over a lifetime, and it is difficult to assess impact on screening rates. Regarding cost figures in Table 3 and Additional file 1: Table S2, the cost per person includes screening, vaccination costs and treatment costs. In addition, these figures are an average cost per person, not just per person vaccinated. Finally, all future costs are discounted, which is why the ICERs are sensitive to discount rate.

\section{Discussion}

We conducted a model-based analysis focusing on routine $9 \mathrm{vHPV}$ vaccination of 12 -year-old girls (70\% coverage) with or without catch-up vaccination (females aged 13-18 years; $30 \%$ coverage).

Our findings indicate that 9vHPV routine plus catchup vaccination of females only yields the most public health benefits (e.g., reductions in HPV-related cervical cancer and genital warts) and may be cost-saving relative to the two alternative options (i.e., screening only and 9vHPV routine vaccination alone) included in this analysis. Sensitivity analyses at assumed vaccination rates of $30 \%$ and $90 \%$ also demonstrated that the model findings were robust to changes in vaccination coverage. Projections of reduced HPV-related disease incidence and cost-effectiveness with routine 9vHPV vaccination and catch-up compared with screening only in this analysis provides a rationale for implementing a regional vaccination program in 12-year-old females in Hong Kong. Given that the ideal scenario is completion of full-course HPV vaccination prior to sexual debut, the low rates of sexual activity among secondary-school students (age 12-18 years; 1-7\%) in Hong Kong [29] further support routine $9 \mathrm{vHPV}$ vaccination in 12 -year-old females.

The cost-effectiveness of catch-up HPV vaccination programs have been previously evaluated, with most studies demonstrating that catch-up vaccination is cost-effective in females aged 12-18 years [30]. However, results regarding cost-effectiveness of routine HPV plus catch-up vaccination programs were inconsistent from these earlier studies, most likely due to differences in aspects of the models used, such as variation in catch-up strategies and comparator strategies, use of herd immunity, differences in time horizons, countryspecific variations (e.g., epidemiology, costs, routine screening practices), and differences in assumptions 
(e.g., calibration, coverage rates, HPV prevalence, sexual activity patterns) [30]. Choi et al. used an age-structured transmission model adapted to estimate the cost-effectiveness and cost-benefit with routine 9vHPV vaccination in Hong Kong for females aged 12 at vaccine uptake rates of $25 \%, 50 \%$, and $75 \%$ [17]. The study demonstrated cost-effectiveness and cost-benefit compared to screening at rates between 25 and $75 \%$ at an estimated upper threshold vaccine cost of USD 444 and USD 689, respectively. However, the analysis only examined benefits of protecting against cervical cancer without accounting for other HPV-related diseases [17]. In contrast, our analysis showed the additional benefits of a catch-up HPV vaccination program.

The current analysis demonstrates that a routine $9 \mathrm{vHPV}$ vaccination program for girls aged 10-12 years with or without catch-up 9vHPV vaccination for females aged 13-18 years is cost-effective. Routine plus catch-up $9 \mathrm{vHPV}$ vaccination is estimated to provide an additional $2.1 \%$ reduction [approximately HKD 61.4 million (USD 7.9 million)] in vaccine-preventable disease costs over routine 9vHPV vaccination alone, resulting in an ICER of HKD 29,911/QALY (USD 3847/QALY). Tay et al. reported similar findings using a validated HPV transmission dynamic model to determine the economic impact of $9 \mathrm{vHPV}$ vaccination over the $4 \mathrm{vHPV}$ and $2 \mathrm{vHPV}$ vaccines in Singapore for females aged 11-12 [19]. The study estimated that 9vHPV vaccination would result in additional overall treatment cost-savings of Singapore dollars (SGD) 61.8 million (USD 46.4 million) and an ICER of SGD 929/QALY (USD 679/QALY), thus highlighting that $9 \mathrm{vHPV}$ vaccination is very cost-effective compared with 2vHPV vaccination [19]. The cost-effectiveness demonstrated in our analysis is also consistent with analyses of other HPV vaccination programs, including those analyzed for the 9vHPV vaccine [18, 19, 31-33].

Our estimates of cost-effectiveness of the 9vHPV vaccine are conservative because our model did not account for indirect costs and, in its current form, only models all nine types found in 9vHPV for cervical and anal cancer. For all other cancers, it models only HPV 16/18. A subgroup analysis of 9vHPV vaccination in Asian participants from two international studies $(\mathrm{N}=2519)$ found that HPV 31/33/45/52/58-related disease was prevented with $>90 \%$ efficacy (efficacy rates of 90.4\%, 94.95\%, $100 \%$, and $100 \%$ in Japan, Thailand, South Korea, and Hong Kong/Taiwan, respectively) [34]. The higher attribution of HPV $52 / 58$ to HPV burden, and in particular among CIN 1/2/3 lesions, in Hong Kong is more likely to be directly addressed with the 9vHPV vaccine, the only available HPV vaccine that targets these HPV types [7]. The higher attribution of HPV 52/58 to cervical disease is also evident in other Asian countries, with higher prevalence rates reported in Asian (i.e., China, Singapore, Japan, Malaysia, Thailand) versus non-Asian (i.e., USA, UK, Australia) countries for HPV 52/58-associated high-grade cervical lesions (HPV 52, 17.4-28.3\% vs 8.910.3\%; HPV 58, 8.2-16.7\% vs 4.9-6.2\%) and cervical cancer (HPV 52, 6.2-10.7\% vs 0.9-2.4\%; HPV 58, 6.2-9.8\% vs $1.1-1.6 \%$ ) [35-43]. Therefore, the external validity of our model may extend beyond Hong Kong to other Asian regions.

\section{Limitations}

A limitation of this study is that it does not account for potential changes to cervical cancer screening with the introduction of routine HPV vaccination. Additionally, there are limited data on the incidence of genital warts in the Hong Kong population because of the variable spectrum of disease manifestation, potential embarrassment for patients, and the transient character of the disease [4]. The model inputs used in this analysis are representative of the cost for all Hong Kong public hospitals, and data gathered are representative of all cases in the Hong Kong population.

The model also assumed no cross-protection against HPV types not targeted by HPV 16/18. Clinical trial results show that the $2 \mathrm{vHPV}$ vaccine provided lower and less consistent protection against HPV types 31/33/45 and lasted for a shorter duration than against directly targeted HPV types (HPV 16/18) [44]. Evidence is also lacking in the real-world setting. Therefore, the crossprotection benefit from 2vHPV would be modest in comparison with the 9vHPV vaccine, which directly targets infection against those HPV types. As a result, the assumption is justified.

\section{Conclusion}

This analysis predicts that the current Hong Kong vaccination strategy can be considered cost-effective and will provide maximum health benefit, with greatest reductions in HPV-related disease incidence compared with all other vaccination strategies considered. Considering the low uptake of cervical screening and the high prevalence of HPV types 52/58 in Hong Kong, preventing HPVrelated disease represents a concern for the Hong Kong government. These results support the incorporation of a catch-up strategy to the current routine 9vHPV female vaccination program for 12-year-old females in Hong Kong.

\section{Abbreviations}

2VHPV: 2-Valent HPV; 9vHPV: 9-Valent human papillomavirus (HPV) vaccine; CIN: Cervical intraepithelial neoplasia; HK: Hong Kong; HKD: Hong Kong dollar; 
HPV: Human papillomavirus; ICER: Incremental cost-effectiveness ratio; QALY: Quality-adjusted life-year; USD: US dollars.

\section{Supplementary Information}

The online version contains supplementary material available at https://doi. org/10.1186/s12962-021-00328-x.

Additional file 1: Table S1. Model Input Variables Implemented in Transmission Dynamic Model. Table S2. Cost-Effectiveness Analysis of the Routine 2vHPV Female-Only Vaccination Strategy vs Screening Only. Table S3. Sensitivity Analyses for Cost-Effectiveness of HPV Vaccination Strategies. Figure S1. Estimated healthcare costs avoided over 100 years by HPV type with routine plus catch-up FOV with the 9vHPV vaccine versus (A) FOV with the $2 \mathrm{VHPV}$ vaccine and (B) FOV with the 9vHPV vaccine.

\section{Acknowledgements}

Medical writing assistance was provided by Lei Bai, PhD, and Maxwell Chang, BSc Hons, of ApotheCom (New York, NY, USA) and was funded by Merck Sharp \& Dohme Corp., a subsidiary of Merck \& Co., Inc., Kenilworth, NJ, USA.

\section{Data sharing}

Merck Sharp \& Dohme Corp., a subsidiary of Merck \& Co., Inc., Kenilworth, NJ, USA's data sharing policy, including restrictions, is available at http://engag ezone.msd.com/ds_documentation.php. Requests for access to the clinical study data can be submitted through the EngageZone site or via email to dataaccess@merck.com.

\section{Authors' contributions}

THC was involved in conception, design or planning of the study, acquisition of the data and interpretation of the results. SSYC was involved in conception, design or planning of the study, acquisition and analysis of the data, interpretation of the results and drafting of the manuscript. DCH was involved in conception, design or planning on the study, and interpretation of the results. QW was involved in analysis of the data and interpretation of the results. AW was involved conception design or planning of the study, analysis of the data, interpretation of the results and drafting of the manuscript. AP was involved in conception, design or planning of the study, analysis of the data and interpretation of the results. KS was involved in analysis of the data and interpretation of the results. VSP was involved in conception, design or planning of the study, analysis of the data interpretation of the results and drafting of the manuscript. All authors contributed to critically reviewing or revising the manuscript. All authors read and approved the final manuscript.

\section{Funding}

This study was sponsored by Merck Sharp \& Dohme Corp., a subsidiary of Merck \& Co., Inc., Kenilworth, NJ, USA.

\section{Availability of data and materials}

All relevant data are contained within the article. The datasets used and/or analyzed in the current study are available from the corresponding author on reasonable request.

\section{Declarations}

\section{Ethics approval and consent to participate}

Not applicable.

\section{Consent for publication}

Not applicable.

\section{Competing interests}

KS, VSP, and AW are employees of Merck Sharp \& Dohme Corp., a subsidiary of Merck \& Co., Inc., Kenilworth, NJ, USA and own stock/stock options for Merck \& Co., Inc., Kenilworth, NJ, USA. DH and QW are employees of Merck Sharp \& Dohme Corp., a subsidiary of Merck \& Co., Inc., Kenilworth, NJ, USA. DH and QW own stock/stock options for Merck \& Co., Inc., Kenilworth, NJ, USA. AP is a consultant for Merck \& Co., Inc. THC and SSYC declared no potential conflicts of interest with respect to the research, authorship, and/or publication of this article.

\section{Author details}

${ }^{1}$ Department of Obstetrics and Gynecology, The Chinese University of Hong Kong, Hong Kong, People's Republic of China. ${ }^{2}$ Global Medical and Scientific Affairs, MSD Asia Ltd, 27/F Lee Gardens Two, 28 Yun Ping Road, Causeway Bay, Hong Kong, People's Republic of China. ${ }^{3}$ MRL, Merck \& Co., Inc., 2000 Galloping Hill Road, Kenilworth, NJ 07033, USA. ${ }^{4}$ HCL America, Inc., Sunnyvale, CA, USA.

Received: 27 January 2021 Accepted: 2 November 2021

Published online: 20 November 2021

\section{References}

1. Bruni L, Albero G, Serrano B, et al. ICO/IARC information centre on HPV and cancer (HPV Information Centre). Human papillomavirus and related diseases: Asia. Summary report. June 17, 2019. https://www.hpvcentre. net/statistics/reports/XSX.pdf. Accessed 19 Jan 2021.

2. Burd EM. Human papillomavirus and cervical cancer. Clin Microbiol Rev. 2003;16(1):1-17.

3. Hong Kong Cancer Registry, Hospital Authority. Cervical cancer in 2017 fact sheet. October 2019. https://www3.ha.org.hk/cancereg/pdf/facts heet/2017/cx_2017.pdf. Accessed 19 Jan 2021.

4. Lin C, Lau JT, Ho KM, Lau MC, Tsui HY, Lo KK. Incidence of genital warts among the Hong Kong general adult population. BMC Infect Dis. 2010;10:272.

5. Munoz N, Bosch FX, de Sanjose S, Herrero R, Castellsague X, Shah KV, et al. Epidemiologic classification of human papillomavirus types associated with cervical cancer. N Engl J Med. 2003;348(6):518-27.

6. Lau YM, Cheung TH, Yeo W, Mo F, Yu MY, Lee KM, et al. Prognostic implication of human papillomavirus types and species in cervical cancer patients undergoing primary treatment. PLOS ONE. 2015;10(4): e0122557.

7. Chan PKS, Cheung TH, Li WH, Yu MY, Chan MYM, Yim SF, et al. Attribution of human papillomavirus types to cervical intraepithelial neoplasia and invasive cancers in southern China. Int J Cancer. 2011:131:692-705.

8. de Martel C, Plummer M, Vignat J, Franceschi S. Worldwide burden of cancer attributable to HPV by site, country and HPV type. Int I Cancer. 2017;141(4):664-70

9. Garland SM, Steben M, Sings HL, James M, Lu S, Railkar R, et al. Natural history of genital warts: analysis of the placebo arm of 2 randomized phase III trials of a quadrivalent human papillomavirus (types 6, 11, 16, and 18) vaccine. J Infect Dis. 2009;199(6):805-14.

10. World Health Organization. Human papillomavirus vaccines: WHO position paper. Geneva: World Health Organization: 2017.

11. Centre for Health Protection. Scientific committee on vaccine preventable diseases and scientific committee on AIDS and STI. Consensus statement on the use of human papillomavirus (HPV) vaccine in prevention of cervical cancer. Published September 2016. https://www.chp.gov.hk/ files/pdf/consensus_statement_on_the_use_of_hpv_vaccine_in_preve ntion_of_cervical_cancer.pdf. Accessed 19 Jan 2021

12. Centre for Health Protection. Department of Health. The Government of the Hong Kong Special Administrative Region. Topical health report No. 4. Prevention and screening of cervical cancer. Published 2004. https:// www.chp.gov.hk/files/pdf/grp-thr-report4-en-20041209.pdf. Accessed 19 Jan 2021

13. Li SL, Lau YL, Lam TH, Yip PS, Fan SY, Ip P. HPV vaccination in Hong Kong: uptake and reasons for non-vaccination amongst Chinese adolescent girls. Vaccine. 2013;31(49):5785-8.

14. Chen JMT, Leung DYP. Factors associated with human papillomavirus vaccination among Chinese female university students in Hong Kong. Am Int J Soc Sci. 2014;3(4):56.

15. Choi HC, Leung GM, Woo PP, Jit M, Wu JT. Acceptability and uptake of female adolescent HPV vaccination in Hong Kong: a survey of mothers and adolescents. Vaccine. 2013;32(1):78-84.

16. The Government of Hong Kong Special Administrative Region. LCQ10: human papillomavirus vaccination programme Hong Kong, China: The Government of Hong Kong Special Administrative Region. Updated 20 Jan 2021. https://www.info.gov.hk/gia/general/202101/20/P202101200 0507.htm. Accessed 22 July 2021. 
17. Choi HCW, Jit M, Leung GM, Tsui KL, Wu JT. Simultaneously characterizing the comparative economics of routine female adolescent nonavalent human papillomavirus (HPV) vaccination and assortativity of sexual mixing in Hong Kong Chinese: a modeling analysis. BMC Med. 2018;16(1):127.

18. Drolet M, Laprise JF, Boily MC, Franco EL, Brisson M. Potential costeffectiveness of the nonavalent human papillomavirus (HPV) vaccine. Int J Cancer. 2014;134(9):2264-8.

19. Tay SK, Hsu TY, Pavelyev A, Walia A, Kulkarni AS. Clinical and economic impact of school-based nonavalent human papillomavirus vaccine on women in Singapore: a transmission dynamic mathematical model analysis. BJOG. 2018;125(4):478-86.

20. Elbasha EH, Dasbach EJ. Impact of vaccinating boys and men against HPV in the United States. Vaccine. 2010;28(42):6858-67.

21. Centers for Disease Control and Prevention. HPV and cancer. Diagnosis by age. Updated 3 Sept 2020. https://www.cdc.gov/cancer/hpv/statistics/ age.htm. Accessed 25 Oct 2021.

22. Census and Statistics Department.The Government of the Hong Kong Special Administrative Region. https://www.censtatd.gov.hk/hkstat/sub/ sp150.jsp?tablelD=002\&ID=0\&productType=8 1. Accessed 19 Jan 2021 .

23. Dasbach EJ, Elbasha EH, Insinga RP. Mathematical models for predicting the epidemiologic and economic impact of vaccination against human papillomavirus infection and disease. Epidemiol Rev. 2006;28:88-100.

24. Gray A, Clarke PM, Wolstenholme JL, Wordsworth S. Applied methods of cost-effectiveness analysis in healthcare. Oxford: Oxford University Press; 2010.

25. Hong Kong Cancer Registry, Hospital Authority. Cancer statistics query systems (CanSQS) —all ages. 2015. https://www3.ha.org.hk/cancereg/ allages.asp. Accessed 19 Jan 2021.

26. Census and Statistics Department. The Government of the Hong Kong Special Administrative Region. Hong Kong annual digest of statistics, 2016 Edition. https://www.statistics.gov.hk/pub/B10100032016AN1 6B0100.pdf. Accessed 19 Jan 2020.

27. The Family Planning Association of Hong Kong. The report of youth sexuality study 2011. Hong Kong: The Family Planning Association of Hong Kong; 2013

28. The Government of the Hong Kong Special Administrative Region. https://gia.info.gov.hk/general/201904/17/P2019041700367_308529_1_ 1555476821983.pdf. Accessed 25 Oct 2021.

29. The Family Planning Association of Hong Kong. Report on youth sexuality study 2016: The Family Planning Association of Hong Kong. Updated 12 July 2017. https://www.famplan.org.hk/en/media-centre/press-releases/ detail/fpahk-report-on-youth-sexuality-study. Accessed 22 July 2021.

30. de Peuter MA, Littlewood KJ, Annemans L, Largeron N, Quilici S. Cost effectiveness of catch-up programs in human papillomavirus vaccination. Expert Rev Vaccines. 2010;9(10):1187-201.

31. Chesson HW, Markowitz LE, Hariri S, Ekwueme DU, Saraiya M. The impact and cost-effectiveness of nonavalent HPV vaccination in the United States: estimates from a simplified transmission model. Hum Vaccin Immunother. 2016;12(6):1363-72.

32. Mahumud RA, Alam K, Dunn J, Gow J. The cost-effectiveness of controlling cervical cancer using a new 9-valent human papillomavirus vaccine among school-aged girls in Australia. PLoS ONE. 2019;14(10): e0223658.

33. Cody P, Tobe K, Abe M, Elbasha EH. Public health impact and cost effectiveness of routine and catch-up vaccination of girls and women with a nine-valent HPV vaccine in Japan: a model-based study. BMC Infect Dis. 2021:21(1):11.

34. Garland SM, Pitisuttithum P, Ngan HYS, Cho CH, Lee CY, Chen CA, et al. Efficacy, immunogenicity, and safety of a 9 -valent human papillomavirus vaccine: subgroup analysis of participants from Asian countries. J Infect Dis. 2018;218(1):95-108.

35. ICO/IARC HPV Information Centre. Australia. Human papillomavirus and related cancers fact sheet. ICO/IARC HPV information centre. Updated 17 June 2019. https://hpvcentre.net/statistics/reports/AUS_FS.pdf?t=16226 28722899. Accessed 22 July 2021.

36. Centre IIHI. Japan. Human papillomavirus and related cancers fact sheet. ICO/IARC HPV information centre. Updated 17 June 2019. https://hpvce ntre.net/statistics/reports/JPN_FS.pdf?t=1557677064251. Accessed 22 July 2021.

37. ICO/IARC HPV Information Centre. United Kingdom. Human papillomavirus and related cancers fact sheet. ICO/IARC HPV information centre. Updated 17 June 2019. https://hpvcentre.net/statistics/reports/GBR_FS. pdf?t=1622628700539. Accessed 22 July 2021.

38. ICO/IARC HPV Information Centre. Canada. Human papillomavirus and related cancers fact sheet. ICO/IARC HPV information centre. Updated 17 June 2021. https://hpvcentre.net/statistics/reports/CAN_FS.pdf?t=16226 28678924. Accessed 22 July 2021

39. ICO/IARC HPV Information Centre. USA. Human papillomavirus and related cancers fact sheet. ICO/IARC HPV information centre. Updated 17 June 2019. https://hpvcentre.net/statistics/reports/USA_FS.pdf?t=16226 28169850. Accessed 22 July 2021

40. ICO/IARC HPV Information Centre. Thailand. Human papillomavirus and related cancers fact sheet. ICO/IARC HPV information centre. Updated 17 June 2019. https://hpvcentre.net/statistics/reports/THA_FS.pdf?t=15576 77645930. Accessed 22 July 2021.

41. ICO/IARC HPV Information Centre. Malaysia. Human papillomavirus and related cancers fact sheet. ICO/IARC HPV information centre. Updated 17 June 2019. https://hpvcentre.net/statistics/reports/MYS_FS.pdf?t=15576 78064474. Accessed 22 July 2021.

42. ICO/IARC HPV Information Centre. Singapore. Human papillomavirus and related cancers fact sheet. ICO/IARC HPV information centre. Updated 17 June 2019. https://hpvcentre.net/statistics/reports/SGP_FS.pdf?t=15576 77890057. Accessed 22 July 2021.

43. ICO/IARC HPV Information Centre. China. Human papillomavirus and related cancers fact sheet. ICO/IARC HPV information centre. Updated 17 June 2019. https://hpvcentre.net/statistics/reports/CHN_FS.pdf?t=15576 76676512. Accessed 22 July 2021

44. Wagner M, Patel H, Badgley D, Joura E, Stanley M, Yen GP, et al. Variable cross-protection effect of HPV vaccine from a systematic literature review. IPVC. Oct 2-6, 2018; Sydney, Australia.

45. The Government of the Hong Kong Special Administrative Region. Cervical screening programme annual statistics report 2014-2015. 2015. http://www.cervicalscreening.gov.hk/english/sr/files/2014 Eng.pdf. Accessed 18 July 2020.

46. Chan PKS, Pong WM, Chan ACL, Chan AKC, Cheung TH, Wong MCS, et al. Distribution of human papillomavirus types in cervical cancers in Hong Kong: current situation and changes over the last decades. Int J Cancer. 2009:125:1671-7.

\section{Publisher's Note}

Springer Nature remains neutral with regard to jurisdictional claims in published maps and institutional affiliations.

Ready to submit your research? Choose BMC and benefit from:

- fast, convenient online submission

- thorough peer review by experienced researchers in your field

- rapid publication on acceptance

- support for research data, including large and complex data types

- gold Open Access which fosters wider collaboration and increased citations

- maximum visibility for your research: over 100M website views per year

At BMC, research is always in progress.

Learn more biomedcentral.com/submissions 